Check for updates

Cite this: RSC Adv., 2019, 9, 39780

\title{
An NIR-triggered drug release and highly efficient photodynamic therapy from PCL/PNIPAm/ porphyrin modified graphene oxide nanoparticles with the Janus morphology $\dagger$
}

\begin{abstract}
Sepideh Khoee (iD * and Amirhossein Sadeghi
This project aimed to investigate the synthesis and characteristics of stimuli-responsive nanoparticles with different morphologies. In the first step, graphene oxide was synthesized based on the improved Hummers' method. Then, thermo-responsive poly( $N$-isopropylacrylamide-co- $N$-(hydroxymethyl)acrylamide), an amphiphilic copolymer, and poly(caprolactone) ( $P C L$ ), a hydrophobic polymer, were used to prepare Janus and mixed graphene oxide-based nanoparticles. Fluorescence microscopy was utilized to confirm the Janus structure by labeling the mixed and Janus NPs with fluorescent hydrophobic and hydrophilic dyes via a solvent-evaporation method. Then, terminally modified carboxyl porphyrin (TPPC3-COOH), used as the second generation photosensitizer, was grafted to the copolymer surrounding the mixed and Janus NPs. Next, quercetin, a hydrophobic anti-cancer drug, was loaded onto both NPs to accomplish NIR-triggered photodynamic- and chemo-therapy. Finally, the drug loading, encapsulation efficiency, and in vitro release of thermo-responsive NPs were investigated at temperatures of $37^{\circ} \mathrm{C}$ and

$40{ }^{\circ} \mathrm{C}$ as well as under laser irradiation $(808 \mathrm{~nm})$.
\end{abstract}

Received 4th August 2019

Accepted 10th November 2019

DOI: $10.1039 / \mathrm{c} 9 \mathrm{ra06058h}$

rsc.li/rsc-advances

\section{Introduction}

Nanomaterials in different sizes, shapes, and chemical compositions, including metal and metal oxide nanoparticles (NPs), polymeric micelles, liposomes, carbon nanotubes, and carbon nanohorns, have emerged as nanocarriers for the delivery of therapeutic agents..$^{1-5}$ During the past decades, the discovery of graphene has attracted considerable attention and growing interest for use in drug delivery applications. Ideal graphene does not exist naturally, but bulk and solution-processable functionalized graphene materials involving graphene oxide (GO) can now be readily obtained. ${ }^{6}$ Graphene oxide, achieved by the chemical oxidation of graphite, benefits from its tendency to disperse in water and physiological environments owing to the presence of numerous hydrophilic groups, such as hydroxyl, epoxy, and carboxyl groups on its basal surfaces, thus providing options for further functionalization by covalent or non-covalent reactions. ${ }^{7}$ Zhang et al. studied the distribution and biocompatibility of GO with an in vivo experimental project and reported that GO was rapidly cleared from the bloodstream and delivered to most of the organs over $48 \mathrm{~h}$, but the agglomeration was mainly in the lungs, liver, and spleen, and to a much lesser extent

Polymer Laboratory, School of Chemistry, College of Science, University of Tehran, PO Box 14155-6455, Tehran, Iran. E-mail: khoee@khayam.ut.ac.ir; Fax: +98 21 66495291; Tel: +982161113301

$\dagger$ Electronic supplementary information (ESI) available. See DOI: $10.1039 / \mathrm{c} 9 \mathrm{ra} 06058 \mathrm{~h}$ in the brain, heart, and bone. The elimination of GO from the body is based on its size. Accordingly, reducing the size of GO particles addresses the issues with its accumulation in the body. ${ }^{8}$ Without any surface functionalization, GO usually tends to aggregate in water and physiological solutions. Thus, size control and particle separation are required to have adequate interactions with a biological system in vitro and in vivo. ${ }^{9-11}$ For instance, drug-loaded GO (GO-DTX) was functionalized via hydrophobic, $\pi-\pi$, or van der Waals interactions with poly(allylamine hydrochloride) (PAH), which is a cationic, highly water-soluble and biocompatible polymer. ${ }^{12}$ Zhang et al. developed a colon-targeted drug delivery system based on alginate-graphene oxide composites. In their studies, GO-ALG/5-FU demonstrated a colon-targeted drug release function, excellent biocompatible properties, and lower drug cytotoxicity. ${ }^{13}$ Recently, dopaminefunctionalized graphene oxide was successfully synthesized and loaded with methotrexate (MTX), followed by drug release studies. ${ }^{14} \mathrm{An}$ interesting type of polymer for use as the coating is a stimuli-sensitive or smart polymer, also called intelligent polymer, due to its response to external triggers, such as changes in light, temperature, or $\mathrm{pH}$, which has widely been studied in various fields, including tissue culture, enzyme immobilization, and drug delivery systems (DDSs). ${ }^{15-18}$ In the past decades, poly $(\mathrm{N}$ isopropylacrylamide) (PNIPAm) has extensively been studied as a thermosensitive polymer in biomedical applications. ${ }^{19}$ The LCST of PNIPAm, which is lower than biological temperatures, can be adjusted to near or above the body temperature by 
copolymerization with hydrophilic monomers, such as acrylic acid (AA). Therefore, by introducing hydrophilic segments in the polymer backbone, a temperature higher than the LCST is needed for hydrophobic interactions..$^{20,21}$ Recently, amphiphilic Janus NPs with bifacial architectural features have appeared as a new kind of colloidal structure to make drug delivery systems more efficient. Various methodologies have been applied to prepare Janus NPs with different functions, such as Pickering emulsion methods, block copolymer self-assembly, microfluidics, and electro co-jetting..$^{22-26}$

Recently, photodynamic therapy (PDT) has become a noticeable protocol in the treatment of cancer and in the inactivation of microorganisms and viruses. ${ }^{27,28}$ In this method a photosensitizer (PS) is introduced into cancer cells irradiating with a laser or visible light, thus generating molecular oxygen. ${ }^{29-31}$ The reactive oxygen species (ROS) generated by photosensitizers can kill cancer cells by destroying DNA, RNA, lipids, and proteins. ${ }^{32}$ However, PDT still has limitations, such as nonspecific tissue biodistribution, insufficient cancer accumulation, rapid renal clearance, and other adverse effects. Porphyrins, a group of organic compounds with a macrocycle conjugated structure, are used as second generation PSs in PDT. Porphyrins and their derivatives exhibit desirable properties, such as selectivity for particular diseased tissues and comparatively fast elimination from the body. However, most porphyrins and their derivatives have hydrophobic properties, making them undispersed and unstable in an aqueous medium, which is a considerable limitation for therapeutic applications. Also, most PSs tend to aggregate in aqueous solution, in which the $\pi$ $\pi$ stacking greatly decreases the yield of ${ }^{1} \mathrm{O}_{2}$ and therefore, the therapeutic efficiency. ${ }^{33}$ To address these issues, much effort has been made to enhance the properties of porphyrin PSs. For example, Zhang et al. prepared reduction-sensitive nanomedicines from porphyrin-containing small molecules and improved the photodynamic therapeutic efficacy at the cellular level. ${ }^{27}$ Li $e t$ al. constructed porphyrin-based carbon dots (TPP CDs) from mono-hydroxylphenyl triphenylporphyrin (TPP) and chitosan, which showed good water solubility and excellent quantum yields of singlet oxygen..$^{34}$

In this work, to control singlet oxygen generation by precise localization and achieve enhanced retention in cancer cells, a system was designed with a more efficient PDT and fewer side effects. To this end, we synthesized mixed and Janus graphene oxide NPs by covalently attaching different types of polymers on their two faces. Poly $(N$-isopropylacrylamide- $c o-N$-(hydroxymethyl)acrylamide), synthesized by the ATRP method, and poly(caprolactone) were used to functionalize GO NPs through a Pickering emulsion of oil in water $(\mathrm{o} / \mathrm{w})$ and a homogeneous solution. To prepare NPs with the ability to generate singlet oxygen $\left({ }^{1} \mathrm{O}_{2}\right)$, these mixed and Janus NPs were decorated with TPPC3-COOH as the second generation photosensitizers. Finally, quercetin, a hydrophobic anti-cancer drug, was loaded in these NPs, and the in vitro release profile of quercetin was studied in a phosphate buffer solution at two temperatures of 37 and $40{ }^{\circ} \mathrm{C}(\mathrm{pH}=7.4)$, as well as under laser irradiation.

\section{Experimental}

\section{Materials}

Graphite flakes, phosphoric acid $\left(\mathrm{H}_{3} \mathrm{PO}_{4}\right)$, potassium permanganate $\left(\mathrm{KMnO}_{4}\right), \mathrm{N}, \mathrm{N}$-dicyclohexylcarbodiimide (DCC), 4-dimethylaminopyridine (DMAP), copper bromide (CuBr), $\varepsilon^{-}$ caprolactone, $\mathrm{N}$-isopropylacrylamide (NIPAm), and $\mathrm{N}$-methylolacrylamide (NMA) were all purchased from Merck Chemical Co. Moreover, $N$-hydroxysuccinimide (NHS), $N$-ethylcarbodiimide hydrochloride (EDC), pyrrole, succinic anhydride, 3-bromo-1-propanol, stannous octoate $\left(\mathrm{Sn}(\mathrm{Oct})_{2}\right)$, and $\mathrm{N}, \mathrm{N}$ dimethylformamide (DMF) were all purchased from Aldrich. All the chemicals were of analytical grade and used without any purification.

\section{Characterization and equipment}

Characterization methods and equipment are reported in the ESI. $\dagger$

\section{Synthesis of graphene oxide}

Graphite flakes were oxidized based on an improved Hummers' method..$^{35}$ Briefly, a mixture of graphite powder $(1 \mathrm{~g})$ in $200 \mathrm{~mL}$ of an acid mixture of $\mathrm{H}_{2} \mathrm{SO}_{4}$ and $\mathrm{H}_{3} \mathrm{PO}_{4}(9: 1, \mathrm{v} / \mathrm{v})$ was prepared under non-stop stirring conditions. Then, $\mathrm{KMnO}_{4}(6 \mathrm{~g})$ was gradually added to the mixture and the reaction was continued for three days at $50{ }^{\circ} \mathrm{C}$. After the completion of the reaction, the mixture was allowed to become cold and then poured into ice water. $\mathrm{H}_{2} \mathrm{O}_{2}(4 \mathrm{~mL})$ was added dropwise to the cold mixture under constant stirring until the color of the solution changed to yellow. Afterward, the mixture was centrifuged and the precipitate was washed twice with an $\mathrm{HCl}$ aqueous solution $(\mathrm{v} / \mathrm{v}$, $10 \%$ ) and three times with distilled water. Dried graphene oxide was obtained after lyophilization.

\section{Synthesis of tert-butyl- $N$-(2-aminoethyl)carbonate (1)}

According to ref. 36, a solution of $13.1 \mathrm{~g}$ (0.06 mol) di-tertbutyldicarbonate in $100 \mathrm{~mL}$ of 1,4-dioxane was gradually poured into a solution of $27.8 \mathrm{~g}(0.46 \mathrm{~mol})$ of diaminoethane in $150 \mathrm{~mL}$ of 1,4-dioxane over $3 \mathrm{~h}$ at room temperature. After $48 \mathrm{~h}$, the precipitate was filtered off and 1,4-dioxane and extra diaminoethane were removed under vacuum. Distilled water $(250 \mathrm{~mL})$ was poured into the residue solution and $\operatorname{bis}\left(N, N^{\prime}\right.$-tertbutyloxycarbonyl)-1,2-diaminoethane was removed by filtration. Saturated sodium chloride was added to the solution and extracted with dichloromethane $(4 \times 100 \mathrm{~mL})$. The organic phase was dried with anhydrous magnesium sulfate, and dichloromethane was evaporated under reduced pressure. Finally, tert-butyl- $\mathrm{N}$-(2-aminoethyl)carbonate was obtained as a colorless oil ( $90 \%$ yield).

\section{Synthesis of tert-butyl-2-(2-bromo-2-methylpropanamido) ethyl carbamate (2)}

tert-Butyl- $N$-(2-aminoethyl)carbonate 1 (3.6 g, $22.50 \mathrm{mmol})$ was dissolved in $40 \mathrm{~mL}$ of THF in the presence of trimethylamine $(3.5 \mathrm{~g}, 33 \mathrm{mmol})$, and then 2-bromoisobutyryl bromide $(7.77 \mathrm{~g}$, 
$33 \mathrm{mmol}$ ) was slowly added to the solution in an ice bath. The reaction solution underwent non-stop stirring at room temperature for $48 \mathrm{~h}$. Triethylammonium bromide as a white precipitate was filtered off and, after the removal of the solvent under vacuum, a yellow solid was obtained which was then dissolved in methanol and precipitated into water saturated with $\mathrm{Na}_{2} \mathrm{CO}_{3}$. Compound 2 was obtained with a $95 \%$ yield. ${ }^{36}$

\section{Synthesis of P(NIPAm-co-NMA)}

The $\mathrm{P}$ (NIPAm-co-NMA) was prepared via atom transfer radical polymerization (ATRP), and 2 was used as an ATRP initiator. First, NIPAm (538.65 mg, $4.76 \mathrm{mmol}$ ), NMA (35.34 mg, 0.35 $\mathrm{mmol})$, and $\mathrm{CuBr}(6.88 \mathrm{mg}, 0.048 \mathrm{mmol})$ were dissolved in methanol/water $(3: 2$, the total volume of solution is $5 \mathrm{~mL}$ ) at room temperature. The initiator $(15.25 \mathrm{mg}, 0.049 \mathrm{mmol})$ and PMDETA $(20.7 \mu \mathrm{L})$ were separately dissolved in $2 \mathrm{~mL}$ of methanol/water $(3: 2)$ and poured into the solution. The solution was purged with nitrogen and heated at $60{ }^{\circ} \mathrm{C}$ for $8 \mathrm{~h}$. To quench the polymerization, the reaction mixture was exposed to air for $2 \mathrm{~h}$. Then, $4 \mathrm{~mL}$ of tetrahydrofuran (THF) was added to the polymerization solution and the final solution was filtered over alumina to remove the catalyst. Next, the solvent was removed by vacuum evaporation and freeze-dried, respectively. $\mathrm{P}(\mathrm{NIPAm}-\mathrm{co}$-NMA) was obtained as a white precipitate and then dissolved in THF and precipitated in hexane. This cycle was repeated in triplicate to achieve a pure product.

\section{Synthesis of poly(E-caprolactone) (PCL)}

According to a previously described method, ${ }^{37}$ poly( $\varepsilon$-caprolactone) was synthesized using ethanol as an initiator, with slight modification. Briefly, a solution of $\varepsilon$-caprolactone $(2 \mathrm{~mL}$, $18.75 \mathrm{mmol}$ ) in DMF ( $5 \mathrm{~mL}$ ) was placed in a three-necked roundbottom flask under nitrogen atmosphere. Then, ethanol (0.15 $\mathrm{mL}, 2.50 \mathrm{mmol}$ ) was poured into the reaction mixture and the temperature was gradually raised to $80^{\circ} \mathrm{C}$. Afterward, a catalytic amount of $\mathrm{Sn}(\mathrm{Oct})_{2}$ was added to the mixture and the solution temperature was adjusted at $120^{\circ} \mathrm{C}$. The reaction medium was allowed to continue at this temperature for $24 \mathrm{~h}$. The raw product was purified by dissolving in DMF and then precipitating in cold water. This cycle was repeated twice to obtain a pure product.

\section{Synthesis of 5-(4-hydroxylphenyl)-10,15,20 triphenylporphyrin (TPP-OH)}

A solution of 4-hydroxybenzaldehyde (780 $\mathrm{mg}, 6.39 \mathrm{mmol})$ in $50 \mathrm{~mL}$ of propionic acid was heated to reflux at $140{ }^{\circ} \mathrm{C}$. Benzaldehyde ( $2 \mathrm{~mL}, 19.74 \mathrm{mmol})$ was then added to the reaction mixture. After $15 \mathrm{~min}$, a solution of pyrrole $(1.74 \mathrm{~mL}, 25.05$ $\mathrm{mmol}$ ) in $5 \mathrm{~mL}$ of propionic acid was added dropwise, and the mixture was refluxed for another $45 \mathrm{~min}$. After cooling to room temperature, $10 \mathrm{~mL}$ of methanol was poured into the mixture and stored in the refrigerator overnight. A purple solid was obtained by washing with cool methanol. The raw product was purified by column chromatography (silica gel) using chloroform as the eluent (yield $=30 \%$ ). ${ }^{38}$

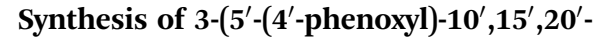
triphenylporphyrin)-1-propanol (TPPC3-OH)

According to ref. 38, with a few changes, TPPC3-OH was synthesized from TPP-OH and 3-bromo-1-propanol. Potassium carbonate (40 mg, $0.29 \mathrm{mmol}$ ) was added to a solution of 3bromo-1-propanol in $5 \mathrm{~mL}$ of DMF. The reaction mixture was refluxed for $16 \mathrm{~h}$ and washed with distilled water three times. The residue was dissolved in $\mathrm{CH}_{2} \mathrm{Cl}_{2}$ and dried with anhydrous $\mathrm{MgSO}_{4}$. Dichloromethane was then removed under vacuum, and the crude product purified with column chromatography (silica gel) using a mixture of petroleum ether-ethyl acetate $(4: 1)$ as the eluent (yield $=90 \%)$.

\section{Synthesis of terminal modified carboxyl porphyrin (TPPC3- $\mathrm{COOH})$}

TPPC3-COOH was synthesized by an esterification reaction between TPPC3-OH and succinic anhydride. Succinic anhydride (520 $\mathrm{mg}, 5.2 \mathrm{mmol}$ ) and pyridine (catalytic amount) were dissolved in $25 \mathrm{~mL}$ of anhydrous methylene chloride at room temperature. Then, DMAP (60 $\mathrm{mg}, 0.49 \mathrm{mmol}$ ) was added to the solution in an ice bath. TPPC3-OH (300 mg, $0.43 \mathrm{mmol}$ ) was mixed in $15 \mathrm{~mL}$ of methylene chloride and added dropwise to the solution. The reaction was performed at $25{ }^{\circ} \mathrm{C}$ for $48 \mathrm{~h}$, and then the solution was washed by water saturated with $\mathrm{NaCl}$ and extracted with dichloromethane. The organic phase was dried with anhydrous $\mathrm{MgSO}_{4}$ and the solvent was removed under vacuum (yield $=90 \%$ ).

\section{Preparation of NPs}

Synthesis of Janus poly( $\varepsilon$-caprolactone)-graphene oxide-poly(NIPAm-co-NMA) (J-(PCL-GO-copolymer)). The Pickering emulsion of the oil-in-water $(\mathrm{o} / \mathrm{w})$ method was used to prepare J(PCL-GO-copolymer). To this end, a solution of PCL $(20 \mathrm{mg}$ ), DCC (8.5 mg), and DMAP (2.5 mg) in $2 \mathrm{~mL}$ DCM as an oil phase was poured dropwise into the homogeneous colloidal suspension prepared beforehand through the dispersion of $10 \mathrm{mg}$ of GO in $10 \mathrm{~mL}$ of phosphate buffer saline ( $\mathrm{pH}$ adjusted to 7.4). The micro-emulsion of $\mathrm{o} / \mathrm{w}$ appeared under a high-rate and nonstop stirring. The first reaction was stopped after $4 \mathrm{~h}$ to confirm the formation of J-(PCL-GO) through several analyses. After that, solid J-(PCL-GO) was dispersed once more in PBS $(10 \mathrm{~mL})$ and sonicated for $30 \mathrm{~min}$ ( $2 \mathrm{~s}$ on/2 $\mathrm{s}$ off) to obtain a homogeneous colloidal suspension of J-(PCL-GO). NHS $(20 \mathrm{mg})$ and EDC (30 $\mathrm{mg}$ ) were added gradually to the reaction solution and stirred for $2 \mathrm{~h}$ at room temperature. Then, poly(NIPAm-co-NMA) (50 $\mathrm{mg}$ ) was added to the reaction, and the mixture was kept at room temperature for $16 \mathrm{~h}$. The aqueous phase was evaporated by the freeze-dryer, and the residue was washed with DCM and then with water to remove unnecessary substances.

Synthesis of mixed poly( $\varepsilon$-caprolactone)-graphene oxidepoly(NIPAm-co-NMA) (m-(PCL-GO-copolymer)). To prepare m(PCL-GO-copolymer), GO (10 mg) was dispersed in DMSO/DMF ( $1: 1$, the total volume is $10 \mathrm{~mL}$ ) through an ultrasound probe (15 min, 45\%). Then, PCL, copolymer, DCC, and DMAP were simultaneously added to the GO suspension. The reaction was 
continued for $24 \mathrm{~h}$ at room temperature. Afterward, the solvent was evaporated through the freeze-dryer, and the product was washed with water and DMF and dried overnight under vacuum.

Functionalization of J-(PCL-GO-copolymer) and m-(PCL-GOcopolymer) by TPPC3-COOH. TPPC3-COOH grafted to Janus and mixed NPs was obtained through the following steps: briefly, J-(PCL-GO-copolymer) (10 mg) was dispersed in $10 \mathrm{~mL}$ of DMSO/DMF (4:1) with the ultrasonic probe (20 $\mathrm{min}, 45 \%$ ). Then, TPPC3-COOH (4.5 mg), the photosensitizer, DMAP (2.5 $\mathrm{mg})$, and DCC ( $8.5 \mathrm{mg}$ ) were added to the colloidal suspension, and the mixture was left to continue for $72 \mathrm{~h}$ under non-stop stirring. Next, the solvent was evaporated by the freeze-dryer. In the final step, the Soxhlet extractor was used for $48 \mathrm{~h}$ to remove the redundant porphyrins, DCC, and DMAP. Mixed(PCL-GO-copolymer) containing porphyrin was also prepared with the same procedure.

\section{Preparation of drug-loaded NPs}

First, $10 \mathrm{mg}$ of the mixed and Janus NPs were separately sonicated in $10 \mathrm{~mL}$ of DMSO/DMF (4:1). Then, quercetin (1 mg), a hydrophobic anti-cancer drug, was added to each colloidal suspension. The mixture was left to continue for $48 \mathrm{~h}$ at room temperature. Drug-loaded NPs were obtained after lyophilization and washed once with the solvent to remove the unloaded drug. In the final step, dried NPs were achieved after freezedrying.

UV-Vis spectroscopy was used to calculate the drug loading and encapsulation efficiency of both NPs. A distinct amount of drug-loaded NP was dispersed in DMSO/DMF ( $4: 1)$ to measure the trapped drug. The solvent mixture of DMSO and DMF easily solvated both PCL and the copolymer and, as a result, the encapsulated quercetin abruptly diffused into the solvent medium. Then, a high-speed centrifuge was employed to separate the insoluble NPs, and the absorbance of quercetin was determined by a UV-Vis spectrophotometer at its maximum absorption wavelength $(370 \mathrm{~nm})$, which was assessed with the help of a pre-determined standard calibration curve of the drug in DMSO/DMF ( $4: 1$ ). The following equations (eqn (1) and (2)) were utilized to measure the drug loading (DL) and encapsulation efficiency (EE):

$$
\begin{aligned}
& \mathrm{DL} \%=\frac{\text { weight of drug in nanoparticles }}{\text { weight of nanoparticles }} \times 100 \\
& \mathrm{EE} \%=\frac{\text { weight of drug in nanoparticles }}{\text { weight of feeding drug }} \times 100
\end{aligned}
$$

\section{In vitro release of quercetin from NPs}

An equal amount of the quercetin-loaded mixed and Janus NPs (1 mg) was separately dispersed in a phosphate buffer saline $(\mathrm{pH}=7.4)$ and transferred to a dialysis bag (molecular weight cut-off of $12 \mathrm{kD}$ ). The bag was placed into a flask containing $50 \mathrm{~mL}$ of fresh PBS $(\mathrm{pH}=7.4)$ and stirred at the rotating speed of $150 \mathrm{rpm}$. The experiment was conducted at both temperatures of 37 and $40{ }^{\circ} \mathrm{C}$. Next, $2 \mathrm{~mL}$ of the release medium was taken at distinct time intervals and replaced with fresh PBS. The amount of released quercetin was calculated through UVVis spectroscopy at the wavelength of $203 \mathrm{~nm}$. The drug release percentage was determined according to eqn (3).

$$
\text { Drug release }(\%)=\frac{M_{\mathrm{t}}}{M_{0}} \times 100
$$

$M_{0}$ and $M_{\mathrm{t}}$ are related to the amount of loaded and released drug, respectively.

\section{Laser irradiation of drug-loaded mixed and Janus NPs}

To evaluate the photothermal effect of NPs, an equal amount of the quercetin-loaded mixed and Janus NPs was irradiated for $20 \mathrm{~min}$ by an $808 \mathrm{~nm}$ continuous-wave NIR laser (power density of $2 \mathrm{~W} \mathrm{~cm}^{-2}$ and spot size of $2 \mathrm{~cm}^{2}$ ). During the NIR exposure, temperature changes were monitored by a $\mathrm{K}$ thermocouple (nickel-chromium) linked to a digital thermometer (Lutron Thermometer TM-917, Taiwan) every $2 \mathrm{~min}$ for $20 \mathrm{~min}$.

\section{Singlet oxygen detection}

A chemical method using ICG (indocyanine green) was applied to assess the capability of the porphyrin-grafted mixed and Janus NPs in singlet oxygen generation. First, a mixture of 80 $\mu \mathrm{L}$ of a colloidal suspension of porphyrin-grafted Janus NPs in DMF $\left(1 \mathrm{mg} \mathrm{mL}^{-1}\right)$ and $10 \mu \mathrm{L}$ of ICG water solution $\left(1 \mathrm{mg} \mathrm{mL}^{-1}\right)$ was added into DMF $(10 \mathrm{~mL})$ and irradiated by a UV light

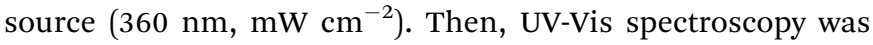
utilized to detect the absorption intensity of the solution every $2 \mathrm{~min}$. The same procedure was replicated for mixed NPs to assess the ability of singlet oxygen generation under irradiation.

\section{Cell cytotoxicity assay}

The relative cell viability was measured in the presence of quercetin, Janus and mixed NPs, quercetin-loaded Janus and mixed NPs, rat C6 glioblastoma and OLN-93 cells (rat brain nontumor cell line) via conventional 3-(4,5-dimethylthiazol-2-yl)2,5-diphenyltetrazolium bromide (MTT) assays. The cells with an initial seeding density of $5 \times 10^{3}$ cells per well were seeded in 96-well plates and cultured for $24 \mathrm{~h}$. For the in vitro phototoxicity of various samples, a cell culture was removed and cells were treated with $100 \mu \mathrm{L}$ fresh medium containing either the above-mentioned samples at the different concentrations for another $24 \mathrm{~h}$. After replacing the fresh culture medium, the cells were exposed to irradiation with a halogen lamp (500-800 nm, $70 \mathrm{~mW} \mathrm{~cm}{ }^{-2}$ ) for $10 \mathrm{~min}$. Then, the plates were incubated for another 24 h. $1 \mathrm{mM}$ freshly-prepared MTT was added to each well and the samples were incubated for $4 \mathrm{~h}$. Then, the culture media were removed and $100 \mu \mathrm{L}$ DMSO was added to each well. Prepared samples were incubated for $10 \mathrm{~min}$. The absorption of the obtained solution was measured at $540 \mathrm{~nm}$. For the dark cytotoxicity, the same procedure and conditions were used but without illumination. 


\section{Statistical analysis}

Statistical analysis of all experiments was done in three independent tests. Results were reported as the mean \pm SD. All statistical analyses were carried using the software Graph Pad Prism 6. The data comparing was performed using a one-way ANOVA. $P<0.05$ shows the statistical significance.

\section{Results and discussion}

\section{Synthesis and characterization of polymer-grafted GO NPs with two morphologies}

This paper presents two methods for the functionalization of GO NPs with two polymers. First, GO NPs were prepared based on the improved Hummer's method. Then, PCL and P(NIPAmco-NMA) were grafted to both or either side of the GO sheets in the absence of emulsifier with two different methods. Finally, mixed and Janus nanoparticles were functionalized with TPPC3$\mathrm{COOH}$ (Scheme 1).

An ultrasonic probe was utilized to decrease the size of GO sheets from millimeters to nanometers. Moreover, GO NPs were synthesized by the oxidation of graphite flakes through the improved Hummer's method (Scheme S1†). The FT-IR spectrum of GO NPs is depicted in Fig. 1a, and the broad peak at $3300-3500 \mathrm{~cm}^{-1}$ was assigned to the stretching vibration of the $\mathrm{OH}$ group. Also, the peaks at 1725, 1626, and $1223 \mathrm{~cm}^{-1}$ were attributed to the $\mathrm{C}=\mathrm{O}, \mathrm{C}=\mathrm{C}$, and $\mathrm{C}-\mathrm{O}$ stretching of the carboxylic, aromatic hydrocarbon, and epoxy groups present on the surface of the GO NPs, respectively.

The synthetic procedures used for the synthesis of P(NIPAmco-NMA) are described in Scheme 2.

The copolymer of NIPAm and NMA was easily prepared by the ATRP method using tert-butyl-2-(2-bromo-2methylpropanamido)ethyl carbamate (2) as the initiator. The ${ }^{1} \mathrm{H}$ NMR spectrum of the initiator shows the methyl proton resonance peaks $\left(\mathrm{s}, 9 \mathrm{H}, \mathrm{C}\left(\mathrm{CH}_{3}\right)_{3}\right.$ at $\delta=1.47 \mathrm{ppm}$ and $\mathrm{s}, 6 \mathrm{H}$, $\mathrm{C}\left(\mathrm{CH}_{3}\right)_{2}$ at $\left.\delta=1.97 \mathrm{ppm}\right)$, along with methylene protons $(\mathrm{q}, 4 \mathrm{H}$, $\mathrm{CH}_{2} \mathrm{CH}_{2}$ ) at $\delta=3.37 \mathrm{ppm}$ and amine protons (s, 2H, NH) at $\delta=$ $4.89 \mathrm{ppm}$ in $\mathrm{CDCl}_{3}$ (Fig. S1 $\dagger$ ). The FT-IR spectra of P(NIPAm-coNMA) are illustrated in Fig. 2A.

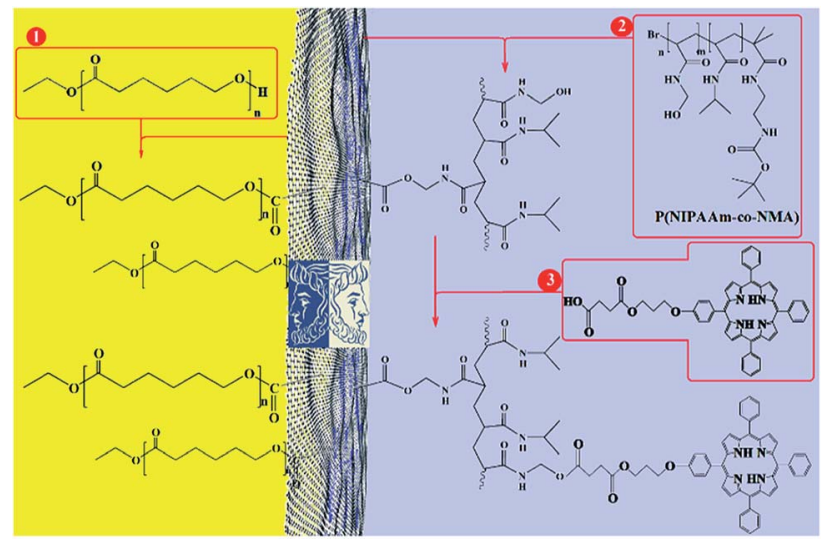

Scheme 1 Total synthesis of porphyrin-decorated Janus poly( $\varepsilon$-caprolactone)-graphene oxide-poly(NIPAm-co-NMA).

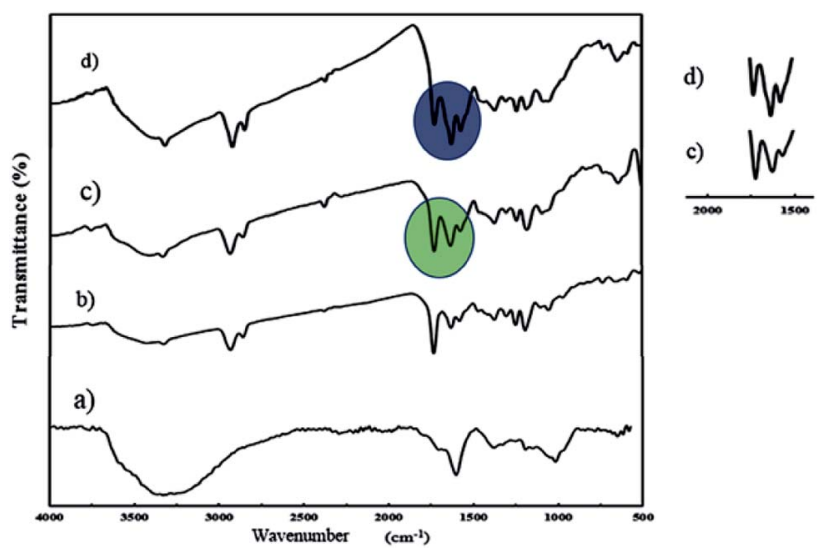

Fig. 1 FT-IR spectra of GO (a), J-(PCL-GO) (b), J-(PCL-GO-copolymer) (c), and $m$-(PCL-GO-copolymer) (d).

The characteristic peaks of the copolymer appearing at 1540 and $1650 \mathrm{~cm}^{-1}$ were attributed to the $\mathrm{C}=\mathrm{O}$ stretching and $\mathrm{N}-\mathrm{H}$ bending of the copolymer, respectively. Furthermore, the successful polymerization of the monomers was confirmed by the disappearance of the absorption band at $1655 \mathrm{~cm}^{-1}$, which are associated with the $\mathrm{C}=\mathrm{C}$ in the vinyl groups. The peaks at 1038 and $3300-3500 \mathrm{~cm}^{-1}$ were assigned to the $\mathrm{C}-\mathrm{O}-\mathrm{H}$ and $\mathrm{O}-\mathrm{H}$ stretching in NMA, respectively. The FT-IR spectrum also shows the main chain absorption band at $2971 \mathrm{~cm}^{-1}$ assigned to the $\mathrm{C}-\mathrm{H}$ stretching bond in the copolymer. The chemical structure of p(NIPAm-co-NMA) was also investigated by ${ }^{1} \mathrm{H}$ NMR spectroscopy in deuterated water $\left(\mathrm{D}_{2} \mathrm{O}\right)$ (Fig. 2B). The signals at $\delta=$ 1.35-2.5 ppm ( $\mathrm{a}$ and $\mathrm{b}$ ) were assigned to the methylene protons $\left(-\mathrm{CH}_{2} \mathrm{CH}-\right)$ of NMA and NIPAm. Furthermore, the peaks at 1.02 (d) and $3.77 \mathrm{ppm}(\mathrm{c})$ were related to NIPAm $\left(-\mathrm{CH}\left(\mathrm{CH}_{3}\right)_{2}\right)$ and NIPAm $\left(-\mathrm{CH}\left(\mathrm{CH}_{3}\right)_{2}\right)$, respectively. The signals at 4.63 (f) and $5.58 \mathrm{ppm}(\mathrm{e})$ were associated with $\left(-\mathrm{NHCH}_{2}-\mathrm{OH}\right)$ and $\left(-\mathrm{CH}_{2} \mathrm{OH}\right)$ for NMA, respectively. ${ }^{39}$ Poly( $N$-isopropylacrylamide) and its

(a)

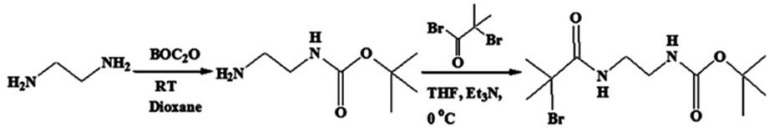

(1)

(2)

(b)

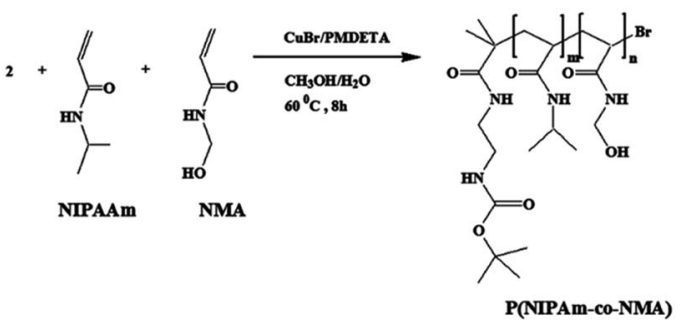

(c)

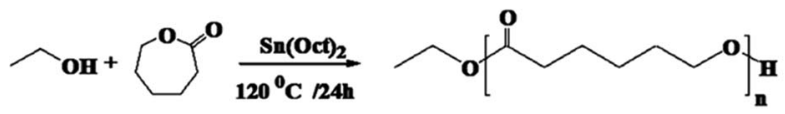

Scheme 2 Synthesis of initiator (a), P(NIPAm-co-NMA) (b), and PCL. 
(A)

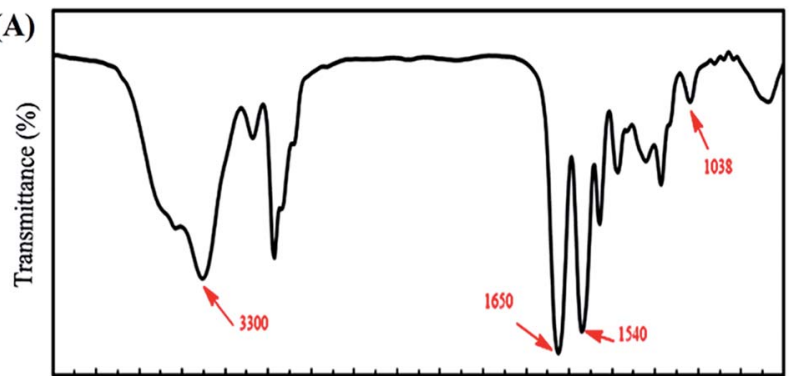

4000380036003400320030002800260024002200200018001600140012001000800600 Wavenumber $\left(\mathrm{cm}^{-1}\right)$

(B)

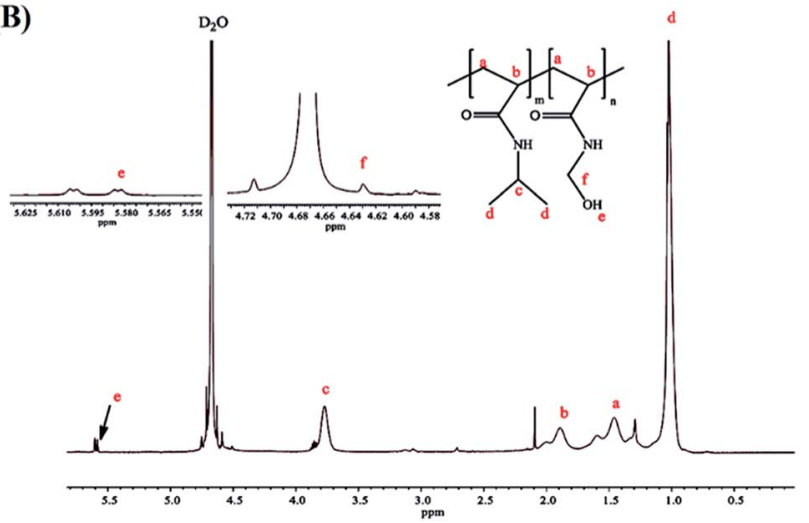

Fig. 2 FT-IR (A) and ${ }^{1} \mathrm{H}$ NMR (in $\mathrm{D}_{2} \mathrm{O}$ ) (B) spectra of poly(NIPAm-coNMA).

copolymers act as thermo-responsive polymers. The LCST of PNIPAm is around $32{ }^{\circ} \mathrm{C}$. It is argued that the LCST of PNIPAm can be adjusted to the body temperature $\left(37^{\circ} \mathrm{C}\right)$ via copolymerization with hydrophilic monomers. ${ }^{40}$ Therefore, poly(NIPAm-co-NMA), with the molar ratio of $(13.5: 1)$, was synthesized to increase the LCST of pure PNIPAm. A UV-Vis spectrophotometer equipped with a temperature controller was utilized to measure the cloud point value of the copolymer solution in water (Table 1). Based on Fig. S2 $\dagger$, the cloud point of poly(NIPAm-co-NMA) shifted to $38.5{ }^{\circ} \mathrm{C}$, indicating that NMA had a significant effect in the copolymer. The molecular weight and the actual NMA content (eqn (4)) in the copolymer were calculated by ${ }^{1} \mathrm{H}$ NMR spectroscopy.

Actual NMA content in the copolymer

$$
=\frac{\text { integration area of peakf } / 2}{\text { integration area of peak } \mathrm{c}+\text { integration area of peak } \mathrm{f} / 2}
$$

The NMA content was estimated by the integration of $\mathrm{CH}\left(\mathrm{CH}_{3}\right)_{2}(3.77 \mathrm{ppm})$ for NIPAm and $\mathrm{NH}-\mathrm{CH}_{2}-\mathrm{OH}(4.63 \mathrm{ppm})$ for NMA. Table 1 presents the detailed information of $\mathrm{p}$ (NIPAmco-NMA).

Poly(\&-caprolactone) was prepared by the ring-opening polymerization of $\varepsilon$-caprolactone in the presence of ethanol and $\mathrm{Sn}(\mathrm{Oct})_{2}$ acting as the initiator and catalyst, respectively, at $120^{\circ} \mathrm{C}$ (Scheme 2C). Fig. S3 $\uparrow$ depicts the FT-IR spectrum of PCL. The characteristic peaks of PCL appeared at 1725 and $1189 \mathrm{~cm}^{-1}$, each associated with the $\mathrm{C}=\mathrm{O}$ and $\mathrm{C}-\mathrm{O}$ stretching bonds of the ester group. Furthermore, the peak at the $2942 \mathrm{~cm}^{-1}$ region was assigned to the $\mathrm{C}-\mathrm{H}$ stretching in the PCL chain. The ${ }^{1} \mathrm{H}$ NMR spectrum of PCL shows six major resonance peaks (Fig. S4 $\dagger$ ). The triplet and multiplet peaks at 0.9 ppm (a) and $4.23 \mathrm{ppm}(\mathrm{b})$ were attributed to the methyl and methylene protons of ethanol, respectively. The methylene protons of the PCL chain appeared at 1.25 (e), 1.7 (d), 2.2 (c), and 4.15 (f) ppm. Moreover, the average molecular weight of PCL is $2000 \mathrm{~g} \mathrm{~mol}^{-1}$, obtained by ${ }^{1} \mathrm{H}$ NMR spectroscopy.

To prepare TPPC3-COOH (Scheme 3), TPP-OH was first synthesized and characterized by ${ }^{1} \mathrm{H}$ NMR (Fig. S5 †). 6-Chloro-1hexanol is the most common spacer for the functionalization of TPP-OH. ${ }^{27,38,41}$ However, in the present work, the hydroxyl group of TPP-OH was expanded to produce TPPC3-OH using 3-bromo1-propanol for the first time because a much shorter spacer affects the solubility of the porphyrin derivatives to a lesser extent. The ${ }^{1} \mathrm{H}$ NMR spectrum of TPPC3-OH is shown in Fig. S6 $†$. The signals at $\delta=8.86-7.25 \mathrm{ppm}$ and $-2.73 \mathrm{ppm}$ were ascribed to the aromatic and pyrrole ring protons separately,

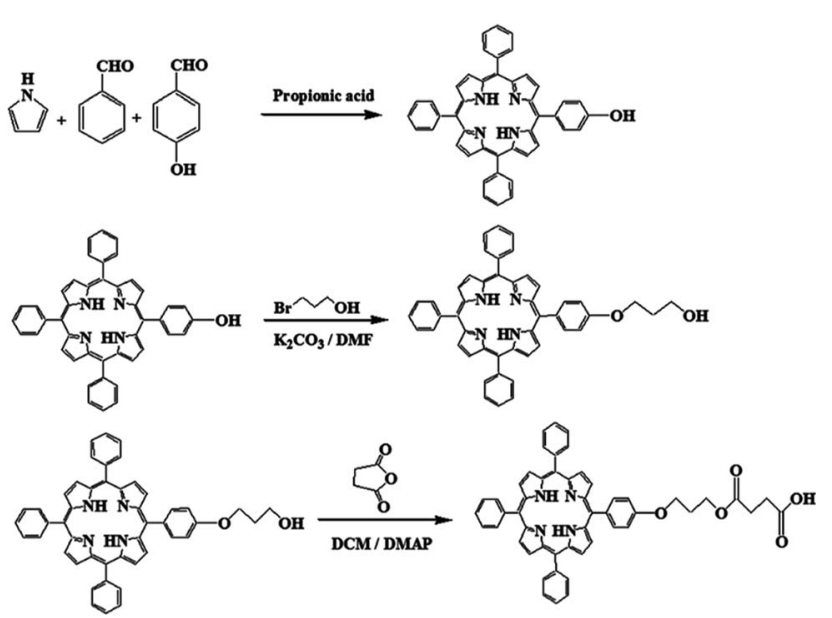

Scheme 3 Synthesis of TPPC3-COOH.

Table 1 Information on the NIPAm-NMA random copolymer

\begin{tabular}{llll}
\hline Sample & $\begin{array}{l}\text { Initial feed molar } \\
\text { ratio (NIPAm : NMA) }\end{array}$ & ${\text { Actual NMA content }{ }^{a}(\%)}$ & $\mathrm{Mn}^{b}$ \\
\hline P(NIPAm-co-NMA) & $13.5: 1$ & 4.76 & 11500
\end{tabular}

${ }^{a}$ The molar content of NMA in poly(NIPAm-co-NMA) determined by ${ }^{1} \mathrm{H}$ NMR. ${ }^{b}$ The molecular weight of the polymer calculated by ${ }^{1} \mathrm{H}$ NMR. ${ }^{c}$ The cloud point measured by UV-Vis spectroscopy. 
and the signals at 4.43, 4.05 and $2.25 \mathrm{ppm}$ were assigned to the methylene protons. Finally, TPPC3-COOH was synthesized for the first time via an esterification between TPPC3-OH and succinic anhydride. The structure of TPPC3-COOH was determined by ${ }^{1} \mathrm{H}$ NMR spectroscopy in $\mathrm{CDCl}_{3}$ (Fig. 3) and a new peak appeared at $\delta=2.75 \mathrm{ppm}$, which belonged to the methylene protons (-CO- $\left.\mathrm{CH}_{2}-\mathrm{CH}_{2}-\mathrm{CO}-\right)$, indicating that TPPC3-COOH was successfully synthesized.

Moreover, the UV-Vis absorption of TPPC3-COOH (Fig. S7 $†$ ) exhibits an intense peak at $420 \mathrm{~nm}$ (Soret band, a characteristic absorption of the electronic spectrum of porphyrin) and a less intense peak at $548 \mathrm{~nm}$ known as the $\mathrm{Q}$ band.

J-(PCL-GO-copolymer) NPs were synthesized with the Pickering emulsion of $\mathrm{o} / \mathrm{w}$ method. $\mathrm{P}$ (NIPAm-co-NMA) possessed amphiphilic behavior and could diffuse into the organic phase. To prevent this problem, J-(PCL-GO-copolymer) NPs were prepared in two steps. Briefly, the oil phase containing the solution of PCL in DCM was added dropwise into the colloidal suspension of GO in phosphate buffer saline (PBS) to obtain J(PCL-GO). The FT-IR spectrum of J-(PCL-GO) NPs (Fig. 1b) has a strong peak at $1728 \mathrm{~cm}^{-1}$, related to the $\mathrm{C}=\mathrm{O}$ stretching bond of the ester group in PCL. In comparison with the FT-IR spectrum of GO NPs (Fig. 1a), the appearance of PCL patterns in the FT-IR spectrum of J-(PCL-GO) NPs confirmed the successful graft of PCL on the surface of the graphene oxide. In the second step, $\mathrm{p}$ (NIPAm-co-NMA) was added to the colloidal suspension of J-(PCL-GO) in the PBS medium to prepare J-(PCL-GOcopolymer) NPs. Thus, there would be a clear boundary between the two polymers grafted on the surface of graphene oxide NPs. The FT-IR spectrum of J-(PCL-GO-copolymer) NPs is shown in Fig. 1c. Compared to J-(PCL-GO), the peaks at 1630 and $1570 \mathrm{~cm}^{-1}$ became stronger, related to the $\mathrm{C}=\mathrm{O}$ stretching and $\mathrm{N}-\mathrm{H}$ bending vibration of the copolymer, respectively.

A one-step reaction was designed to synthesize mixed (PCLGO-copolymer) NPs. Graphene oxide was sonicated in a mixture solvent of DMSO/DMF $(1: 1)$ and then both polymers were simultaneously added to the suspension. In this way, PCL and $\mathrm{p}$ (NIPAm-co-NMA) were haphazardly grafted on both sides of the graphene oxide NPs. The FT-IR spectrum of mixed NPs (Fig. 1d) illustrates that all the characteristic peaks belonging to the functional groups grafted on the surface of graphene oxide

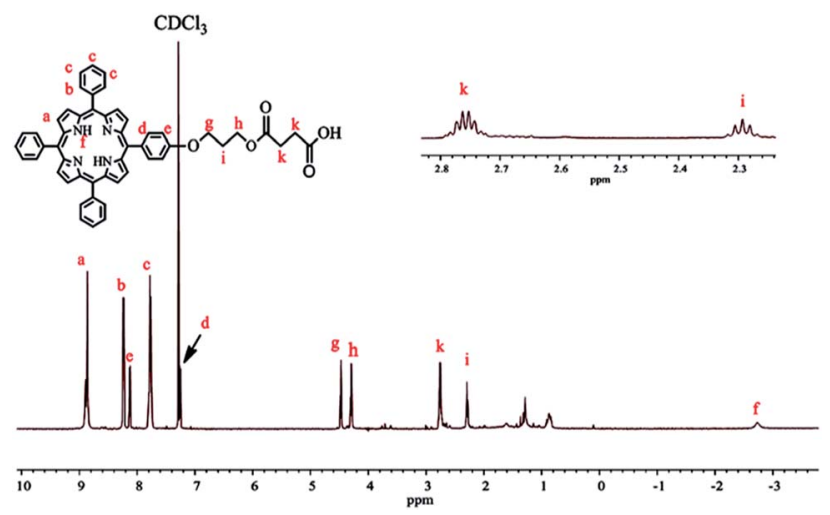

Fig. $3{ }^{1} \mathrm{H}$ NMR spectrum of TPPC3-COOH in $\mathrm{CDCl}_{3}$.

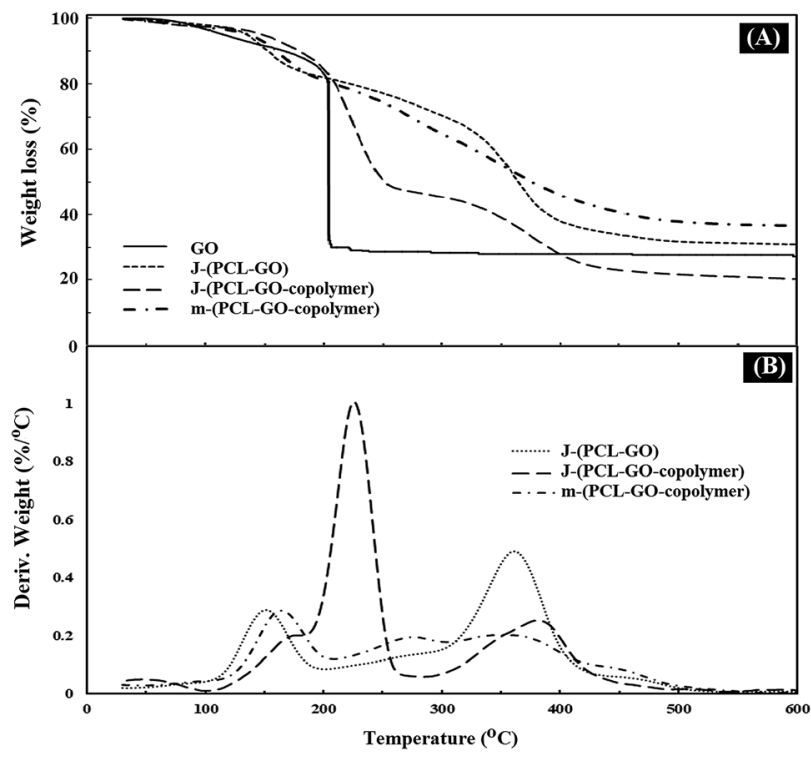

Fig. 4 TGA (A) and DTG (B) thermograms of GO, J-(PCL-GO), J-(PCLGO-copolymer), and m-(PCL-GO-copolymer).

NPs are present. Compared to the Janus NPs, the weak peak at $1728 \mathrm{~cm}^{-1}$ (related to the $\mathrm{C}=\mathrm{O}$ stretching vibration in PCL) appeared in the spectrum of mixed NPs, attributed to the interactions between PCL and copolymer. However, the peaks at 1728 and $1631 \mathrm{~cm}^{-1}$ in the Janus spectrum become more distinct and stronger than the identical peaks in the spectrum of mixed NPs, demonstrating that the PCL-copolymer interactions are not significant in the Janus NPs.

TGA analysis was used to measure the percentage of polymers grafted on the surface of the mixed and Janus NPs. The weight loss curves of GO (a), J-(PCL-GO) (b), J-(PCL-GOcopolymer) (c), and m-(PCL-GO-copolymer) (d) are presented in Fig. 4. Pure graphene oxide showed a weight loss of about $67 \%$, related to the degradation of unstable oxygen-containing functional groups as well as water evaporation. The weight loss curve of J-(PCL-GO) (Fig. 4A and B) depicted two distinct regions. The first mass loss $(18 \%)$ was observed before $200{ }^{\circ} \mathrm{C}$, which is related to the degradation of GO, and the second one
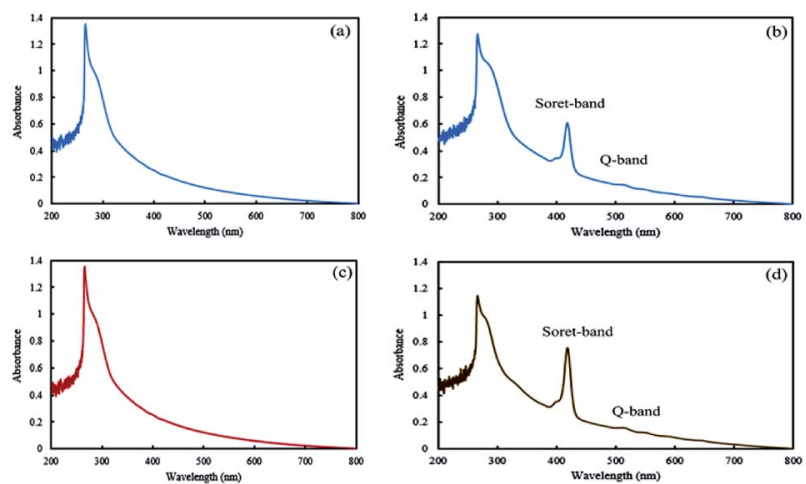

Fig. 5 UV-Vis absorption spectra of mixed ( $a$ and b) and Janus (c and d) NPs before and after TPPC3- $\mathrm{COOH}$ grafting. 

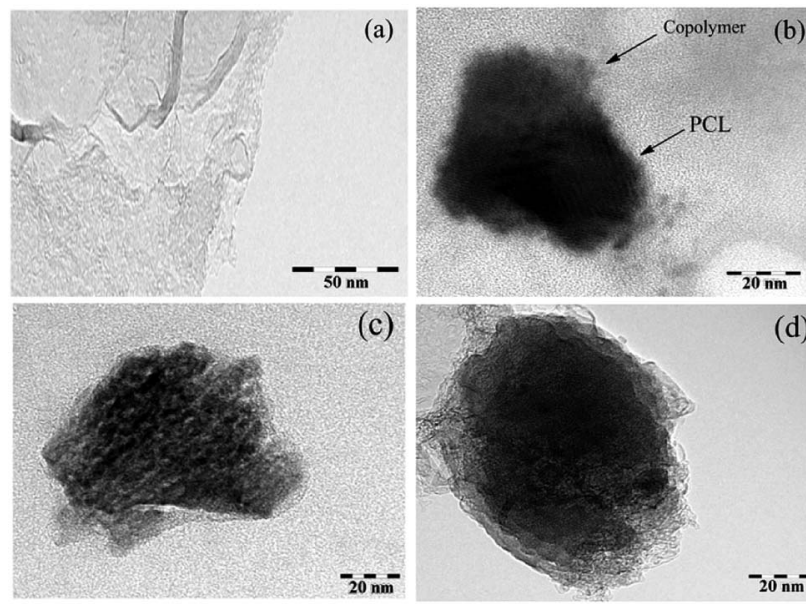

(c)

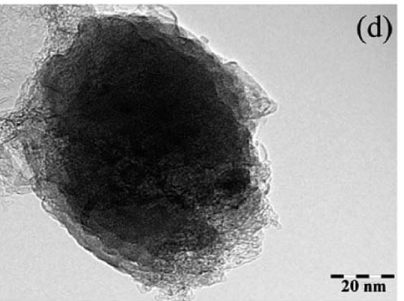

Fig. 6 TEM micrograph of GO (a), J-(PCL-GO-copolymer) (b), and m(PCL-GO-copolymer) (c and d) (at a magnification of 200, 150, 250 and $300 \mathrm{k} \times$, respectively).

(50\%) appeared after $220{ }^{\circ} \mathrm{C}$, which is related to the decomposition of PCL grafted onto the surface of J-(PCL-GO) NPs. According to the TGA curves, J-(PCL-GO-copolymer) NPs gradually lost $15 \%$ of their total weight before $200{ }^{\circ} \mathrm{C}$, which is attributed to the destruction of GO. Then, a weight loss of about $40 \%$ was observed from 200 to $290{ }^{\circ} \mathrm{C}$, belonging to the decomposition of $\mathrm{p}$ (NIPAm-co-NMA). Finally, the third weight loss $(25 \%)$ from 310 to $600{ }^{\circ} \mathrm{C}$ was attributed to the degradation of PCL, verifying the composition of the J-(PCL-GO-copolymer) NPs. m-(PCL-GO-copolymer) NPs lost $20 \%$ of their total weight before $200{ }^{\circ} \mathrm{C}$, which is attributed to the destruction of unstable functional groups on the surface of the graphene oxide. Then, a mass loss of $15 \%$ appeared from 210 to $300{ }^{\circ} \mathrm{C}$, related to the degradation of the copolymer on the mixed NPs. Finally, a larger weight loss of about $28 \%$ was observed from 310 to $600{ }^{\circ} \mathrm{C}$,
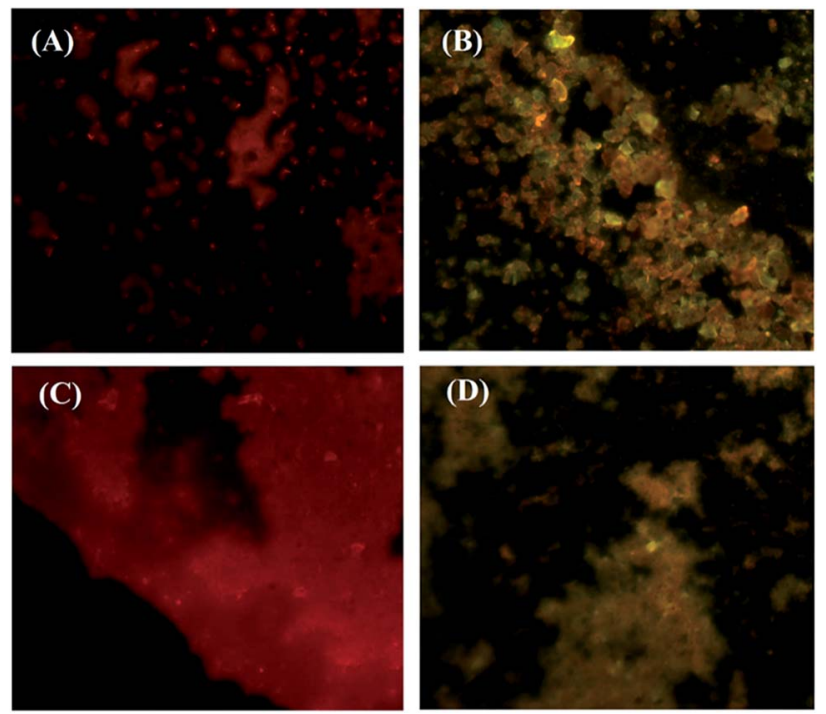

Fig. 7 Fluorescent images of Janus ( $A$ and $B$ ) and mixed ( $C$ and $D)$ nanoparticles under violet ( $A$ and $C$ ) and blue ( $B$ and $D)$ beams.
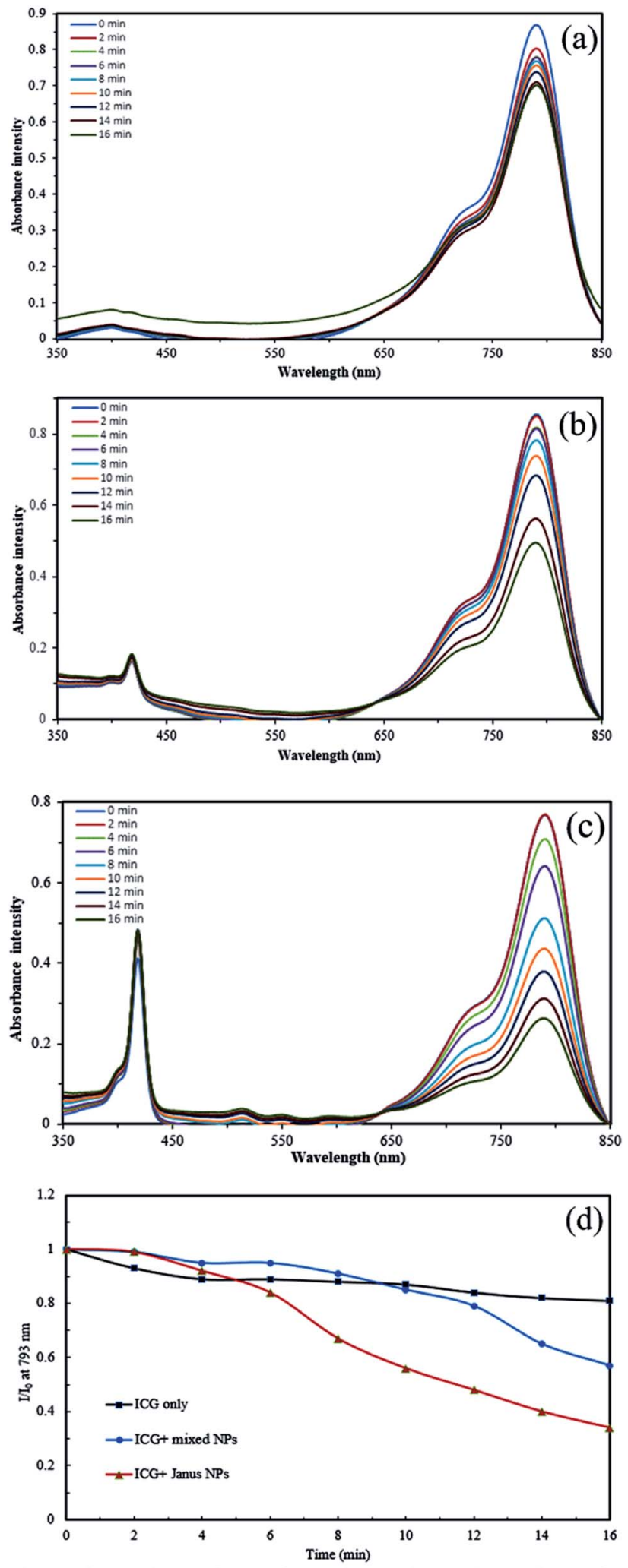

Fig. 8 Time-dependent absorption spectra of (a) ICG alone, (b) ICG in the presence of porphyrin-grafted $\mathrm{m}$-(PCL-GO-copolymer) NPs, (c) ICG in the presence of porphyrin grafted J-(PCL-GO-copolymer) NPS, and (d) decomposition rate of ICG alone, ICG in the presence of the mixed and Janus NPs under irradiation. 
Table 2 Physical properties of NPs with and without drug

\begin{tabular}{|c|c|c|c|c|}
\hline \multirow[b]{2}{*}{ Nanoparticles } & \multicolumn{2}{|l|}{ Janus } & \multicolumn{2}{|l|}{ Mixed } \\
\hline & Without drug & With drug & Without drug & With drug \\
\hline Drug loading (\%) & - & 6.5 & - & 7.9 \\
\hline Encapsulation efficiency (\%) & - & 72.3 & - & 86.9 \\
\hline Size $(\mathrm{nm})$ & $175 \pm 4.6$ & $179.9 \pm 3.58$ & $152 \pm 3.36$ & $161 \pm 5.08$ \\
\hline
\end{tabular}

which is attributed to the decomposition of PCL in the sample. The TGA measurement of J-(PCL-GO-copolymer) NPs in comparison to that of the mixed NPs showed a distinct gap between the decomposition of PCL and copolymer due to the lack of interactions between them in the Janus NPs. This observation is consistent with the findings of previous scientists who demonstrated that this two-stage weight loss is more clearly defined for the block copolymers with micro-phaseseparated domains in comparison that of random ones. ${ }^{\mathbf{4 2}}$ Also, the TGA curves demonstrated that the mixed NPs have a higher thermal stability than that of the Janus ones, and additional energy is required for the degradation of furthermixed NPs. The reason for this is that the polymer-polymer interactions in the mixed NPs lead to a lower chain mobility and higher hydrogen bonding. Moreover, no distinct peaks were observed for the two polymers in the mixed sample.

In the final step of preparing the mixed and Janus NPs, the TPPC3-COOH photosensitizer was grafted to the existing copolymers in J-(PCL-GO-copolymer) and m-(PCL-GOcopolymer) (Scheme 1). After the removal of the redundant TPPC3-COOH using the Soxhlet extractor, a UV-Vis spectrophotometer was utilized to confirm the successful attachment of TPPC3-COOH to poly-(NIPAm-co-NMA) in the mixed and Janus NPs. Before grafting TPPC3-COOH to the NPs, the UV-Vis absorption of the mixed and Janus NPs displayed a peak at $270 \mathrm{~nm}$, which is related to graphene oxide (Fig. 5a and b), while an intense peak placed at $420 \mathrm{~nm}$ (Soret band) and a less intense peak at $530 \mathrm{~nm}$ (Q band) were observed in the absorption spectrum of the TPPC3-COOH grafted mixed and Janus NPS (Fig. 5c and d).

The morphology of the final mixed and Janus NPs was investigated by transmission electron microscopy (TEM) and scanning electron microscopy (SEM). TEM images illustrated in Fig. 6 revealed different morphologies for GO, mixed, and Janus NPs. According to Fig. 6a, the GO sheets had flat surfaces and wrinkled edges. J-(PCL-GO-copolymer) NPs depicted a phase separation. In this case, PCL and copolymer chains were separately grafted on the GO surface with a regular pattern and distinct boundary (Fig. 6b). Nevertheless, in the mixed NPs, polymers were grafted haphazardly with an irregular pattern (Fig. 6c and d). These results confirm that the Pickering emulsion-free emulsifier method had been applied successfully to produce Janus NPs, where GO acted as the surfactant.
The different morphologies of the synthetic NPs were also revealed through SEM. Janus NPs displayed a compact agglomeration (Fig. S8a $\dagger$ ), whereas a separated morphology appeared for the mixed ones (Fig. S8b †). There are certain reasons why Janus NPs adopt such behavior. First, the hydrophobic interactions of the PCL chains lead to the self-assembly of NPs, making up a micro-sized core. ${ }^{43}$ Second, the copolymer chains of several Janus NPs settle on the periphery of the macrocluster to optimize the repulsive interactions.

Fig. 7 shows fluorescence microscopy images of Janus (A and B) and mixed (C and D) nanoparticles irradiated with violet (380-450 $\mathrm{nm}$ ) and blue (450-495 $\mathrm{nm}$ ) beams. As can be seen from the images, the green and red colors are related to fluorescein and acridine orange, respectively, which are located in
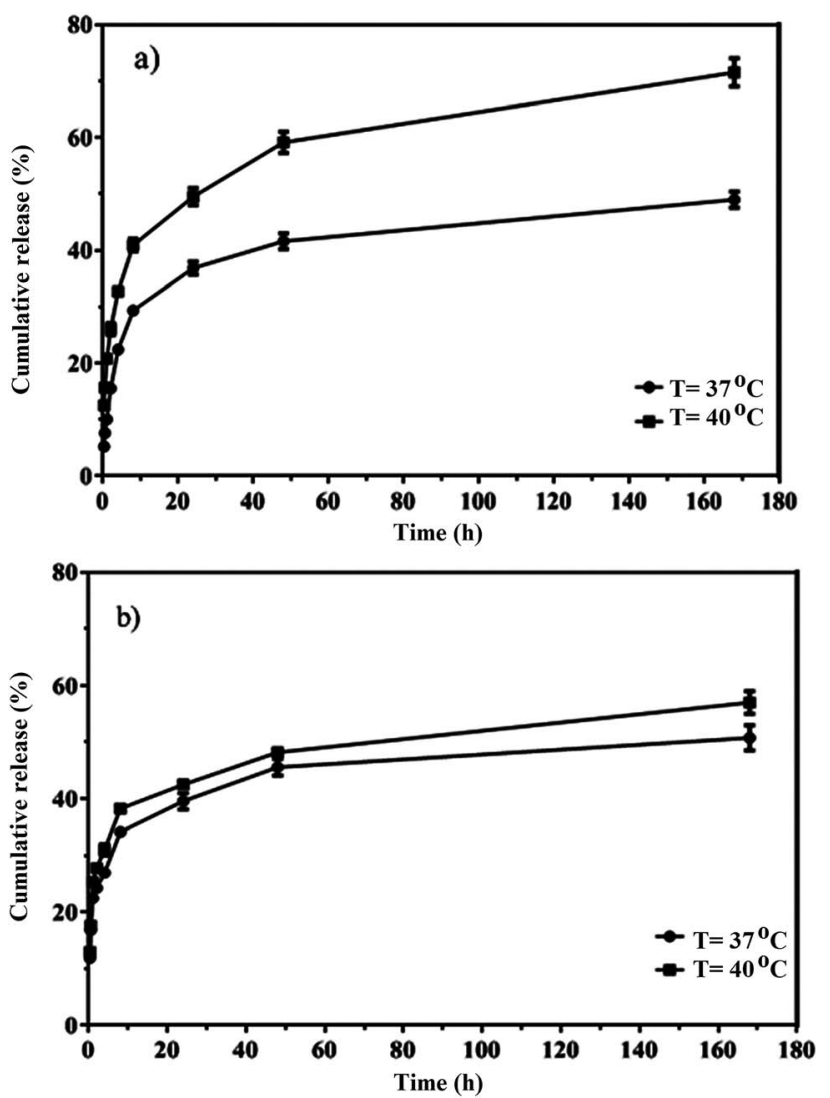

Fig. 9 In vitro release of quercetin from porphyrin-grafted Janus (a) and mixed (b) NPs. 
both NPs. According to Fig. 7A, some brilliant red domains are observable, which are related to the excited acridine concentrated in a half portion of the Janus NPs. However, these Janus NPs under a blue beam show both colors in separated domains (Fig. 7B). In contrast, when the mixed NPs were irradiated with blue and violet beams, a scattered red pattern was illustrated under the violet light due to the presence of acridine (Fig. 7C). The appearance of several borderless domains in red and green colors under the blue light irradiation confirmed the validity of the synthesis of mixed NPs (Fig. 7D).

\section{The evaluation of ${ }^{1} \mathrm{O}_{2}$ generated by porphyrin-grafted Janus and mixed NPs}

The ability of UV-light irradiated NPs to produce singlet oxygen was studied with the help of indocyanine green (ICG) as a probe and time-dependent absorption spectroscopy. Fig. 8 depicts the UV absorption spectra of ICG blank and Janus and mixed NPs in the presence of ICG under irradiation. Based on Fig. 8a, the ICG absorption at $793 \mathrm{~nm}$ under irradiation had a small decrease. However, the absorbance of ICG in the presence of the porphyrin-grafted mixed and Janus NPs under irradiation had a steady decrease over time, which is related to the decomposition of ICG by ${ }^{1} \mathrm{O}_{2}$ generation under irradiation (Fig. 8b and c). In addition, Fig. 8d presents a comparison between the decomposition rates of ICG alone and ICG in the presence of both NPs under irradiation, in which $I_{0}$ and $I$ are the ICG absorbance at $793 \mathrm{~nm}$ in the initial time $(t=0)$ and various times, respectively. ICG absorption in the ICG blank maintained $80 \%$ of its initial absorption, while in the mixed and Janus NPs, it reached $57 \%$ and $34 \%$, respectively. The reason why Janus NPs produce more ${ }^{1} \mathrm{O}_{2}$ than the mixed ones is related to the amount of copolymer grafted on the surface of the GO NPs. According to the TGA curves of the mixed and Janus NPs, the percentage of the copolymer in the Janus NPs is more than that of the mixed ones. As a result, there is more TPPC3-COOH grafted to the Janus NPs. Therefore, the decomposition rate of ICG is effectively higher in the presence of Janus NPs than in the mixed ones.

\section{Preparation and investigation of drug-loaded Janus and mixed NPs}

Quercetin, a hydrophobic anti-cancer drug with two benzene rings, was used for loading into both NPs through the drugtrapping method. Table 2 gives the percentages of drug loading and the encapsulation efficiency of both Janus and mixed NPs with a $10 \mathrm{wt} \%$ drug-to-NP ratio. According to the results of UV-Vis spectroscopy, the mixed NPs have a higher EE\% than that of the Janus ones. Quercetin has the ability to aggregate in the hydrophobic parts of NPs because of its hydrophobic nature. Moreover, it is capable of forming hydrogen bonds with the hydrophilic parts of the NPs. It seems that the interface of PCL (hydrophobic section) and copolymer (amphiphilic section) is the best position for the loading of quercetin. As a result, Janus NPs with fewer hydrophilic/ hydrophobic interfaces depicted a lower DL and EE\% than that of the mixed ones. Dynamic light scattering (DLS) data showed that the mean hydrodynamic diameter of the NPs after quercetin loading increased in a water solution. Although the zeta potential of the drug-loaded NPs declined, the colloidal suspension of NPs had desirable stability (Table 2).

The in vitro release of quercetin from the mixed and Janus NPs was conducted in PBS at 37 and $40{ }^{\circ} \mathrm{C}$ and a $\mathrm{pH}$ value of 7.4. Thermo-sensitive polymers were designed to release the loaded drug at the temperature of cancerous cells (above $37^{\circ} \mathrm{C}$ ). The drug release profiles of the mixed and Janus NPs were calculated at 37 (body temperature) and $40{ }^{\circ} \mathrm{C}$ to assess the sensitivity of these NPs. Based on Fig. 9, the release curves of both types of NPs were higher at $40{ }^{\circ} \mathrm{C}$ than $37^{\circ} \mathrm{C}$. In addition, compared to our previous work, ${ }^{\mathbf{4 4}}$ Janus NPs were more sensitive to temperature changes than the mixed ones due to the lower interaction between PCL and copolymer in the Janus NPs.

Graphene and graphene oxide are capable of converting NIR radiation into vibrational energy and heat. ${ }^{45}$ As shown in Fig. 10A, the temperature of J-(PCL-GO-copolymer) and m-(PCLGO-copolymer) NPs increased rapidly from room temperature to 70.9 and $86.3{ }^{\circ} \mathrm{C}$, respectively. In comparison, there were no significant changes in the temperature of quercetin alone under NIR laser radiation. These results show that the synthesized
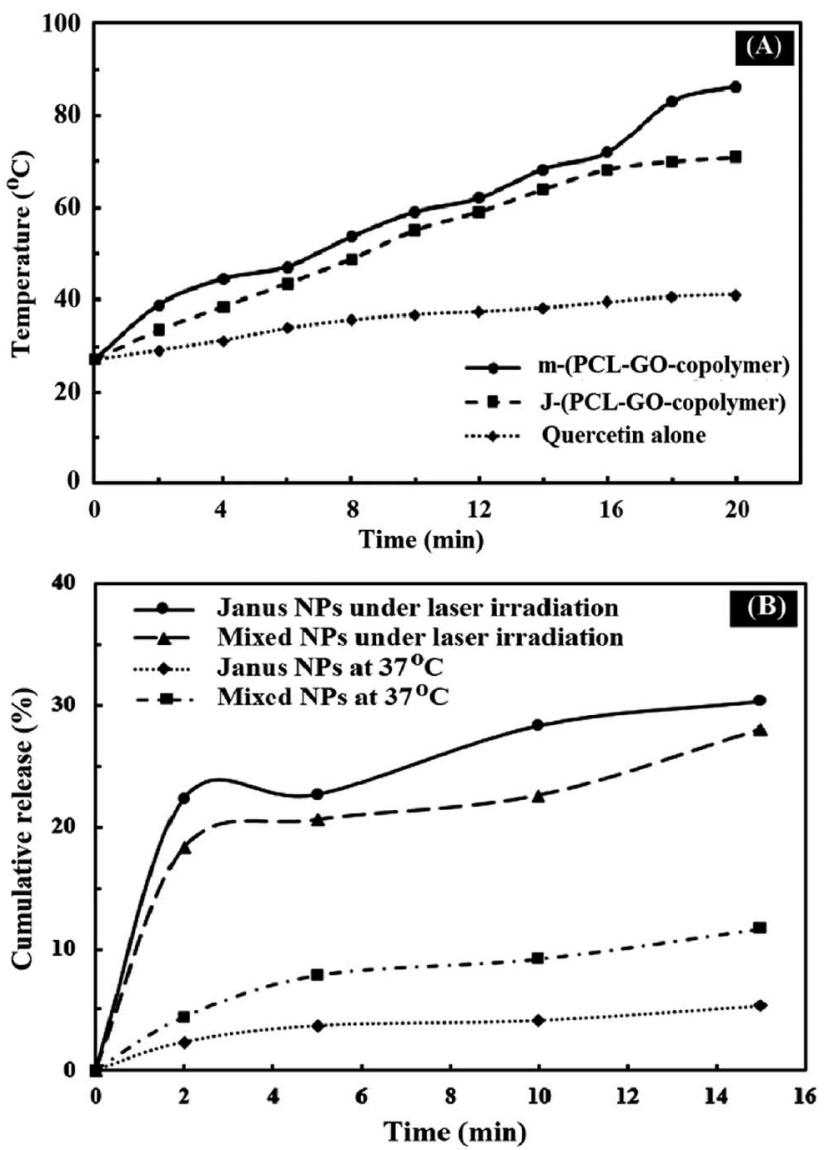

Fig. 10 Photothermal capacity of quercetin, mixed and Janus NPs (A) under irradiation with $808 \mathrm{~nm}$ laser for $20 \mathrm{~min}$; NIR-triggered release of quercetin from porphyrin-grafted Janus and mixed NPs with a temperature change under NIR irradiation $\left(2 \mathrm{~W} \mathrm{~cm}^{-2}\right)$ for $15 \mathrm{~min}$ and at $37^{\circ} \mathrm{C}$ using a heater stirrer for $15 \mathrm{~min}(\mathrm{~B})$. 

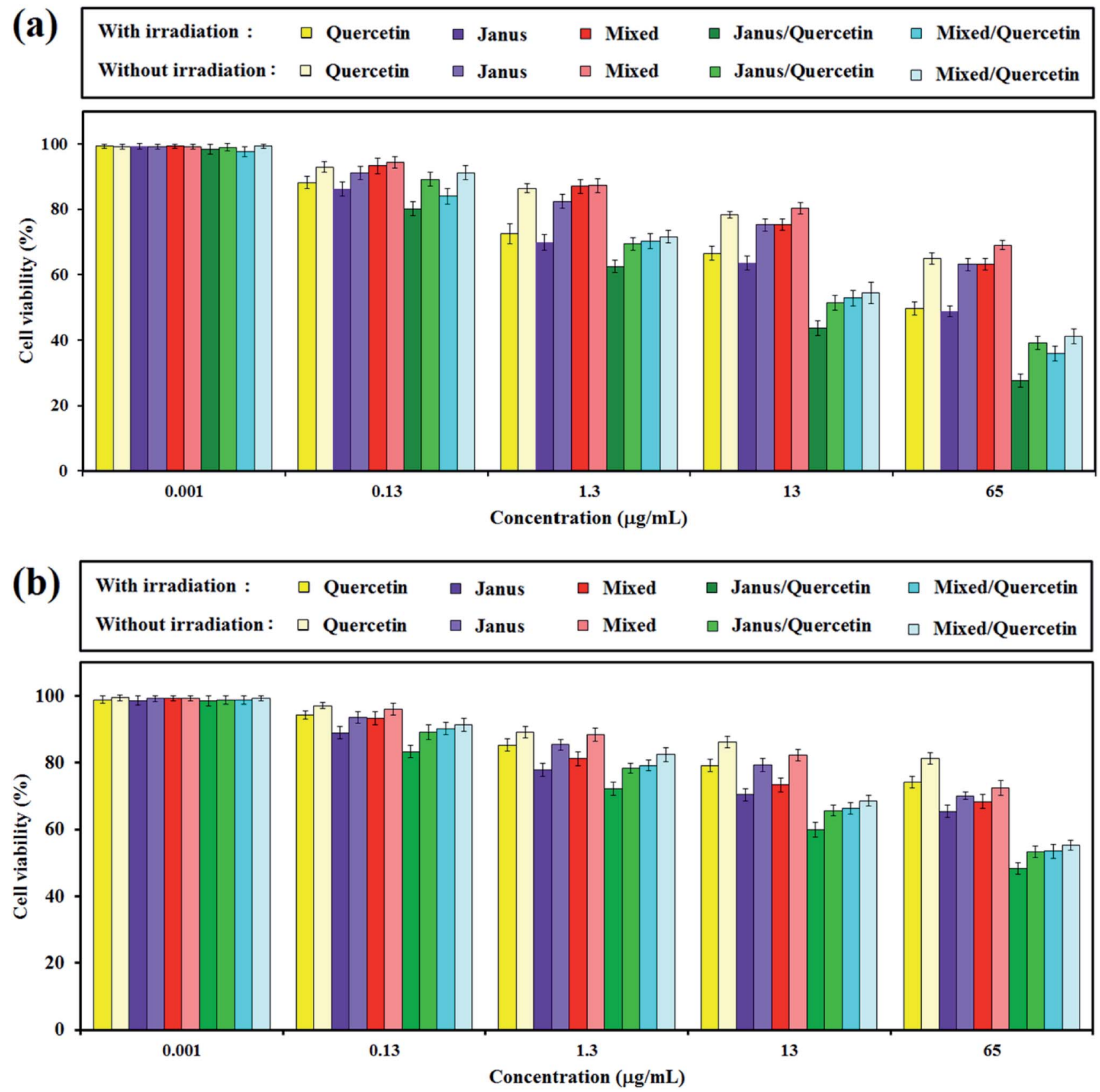

Fig. 11 Viability of C6 glioblastoma (a) and OLN-93 (b) cells evaluated by the MTT assay with (10 min) and without light illumination after treating with quercetin, mixed and Janus NPs, and quercetin-loaded mixed and Janus NPs at different concentrations.

mixed and Janus NPs can be used effectively as a photothermal agent in photothermal therapy. In addition, the accelerated quercetin release of the drug-loaded mixed and Janus NPs was measured through the photothermal effect of the Janus and mixed NPs under NIR laser irradiation $(808 \mathrm{~nm}$, power density of $2 \mathrm{~W} \mathrm{~cm}^{-2}$, and spot size of $2 \mathrm{~cm}^{2}$ ). Based on Fig. 10B, a burst release of quercetin was observed from the Janus and mixed NPs in 15 min, which is significantly higher than the cumulative release of quercetin from these NPs at $37^{\circ} \mathrm{C}$ at the same time without NIR laser irradiation. Also, it is clear that the Janus NPs were more effective than the mixed ones because the release profile of the Janus NPs at $37{ }^{\circ} \mathrm{C}$ was lower than that of the mixed ones without laser irradiation, while the release profile of quercetin in the Janus NPs significantly increased under laser irradiation. These results indicate that the NIR irradiation on NPs was transformed into vibrational energy to produce sufficient heat to increase the release profile of quercetin in both NPs.

\section{Cytotoxicity tests}

The in vitro cytotoxicity of free quercetin, mixed and Janus NPs, as well as quercetin-loaded mixed and Janus NPs with and 
without light illumination, were tested via an MTT assay against C6 glioblastoma and OLN-93 cells. Fig. 11a shows the viability of the incubated cancerous cells with the mentioned samples for $24 \mathrm{~h}$. It is clear that the porphyrin-grafted mixed and Janus NPs exhibited a low dark cytotoxicity, even at a maximum concentration of $65 \mu \mathrm{g} \mathrm{mL}^{-1}$, and they could be safely used for drug delivery systems. In comparison with empty NPs, the quercetinloaded mixed and Janus NPs show more dark toxicity, especially at higher concentrations.

The phototoxicity of the mentioned samples was then confirmed via light irradiation with a halogen lamp (500$800 \mathrm{~nm}, 70 \mathrm{~mW} \mathrm{~cm}^{-2}$ ) for an irradiation time of $10 \mathrm{~min}$. According to Fig. 11a, the smaller phototoxicity of the mixed NPs compared to the Janus ones might correlate with the lower grafting of porphyrin in the mixed NPs, which caused insufficient photo-activity and generates less singlet oxygen. In addition, the quercetin-loaded mixed and Janus NPs exhibit potential phototoxicity over all samples. The results indicate that the synergistic effect of a combined chemotherapeutic drug and PDT is acceptable and can be considered as a favourable result. The phototoxicity of quercetin-loaded Janus NPs against cancerous cells was about identical to other reported work with nanoparticles containing $\pi-\pi$ interactions between GO and porphyrin. ${ }^{45}$

The inhibitory effect of different nanoparticles on cell proliferation of OLN-93 as normal cells was performed similarly to those for C6 cells. According to Fig. 11b, the dark toxicity of quercetin-free Janus and mixed NPs on normal and cancerous cells is approximately identical. Quercetin was more toxic to cancerous cells compared to normal cells in dark conditions. ${ }^{46}$ When the concentration of NPs increased, the cell viability of OLN-93 dwindled steadily, especially when irradiated by the halogen lamp. And yet, the inhibition of cancer cell proliferation was greater than those of normal ones.

\section{Conclusions}

In this work, we synthesized a thermo-responsive NIPAm-based copolymer by the ATRP method and a biocompatible PCL through a ring-opening polymerization. An amphiphilic copolymer and hydrophobic PCL were used to prepare polymermodified graphene oxide NPs with mixed and Janus morphologies. Two-face Janus NPs were prepared with the Pickering emulsion method of o/w. Then, TPPC3-COOH was grafted onto the copolymer-grafted mixed and Janus NPs. We studied the different characteristics of quercetin-loaded NPs, such as size, morphology, drug capacity, and in vitro release. It was found that Janus NPs exhibit different behaviors in the TGA curves and TEM images. Furthermore, mixed NPs depict a higher quercetin loading than the Janus NPs due to the higher number of polymer-polymer boundaries in the mixed NPs, which is a suitable environment for trapping quercetin. However, the drug release studies revealed that Janus NPs are more sensitive to temperature changes from 37 to $40{ }^{\circ} \mathrm{C}$, making this NP suitable for drug delivery systems in the human body. Also, the in vitro release of quercetin under laser irradiation was studied and the results showed that the drug release profile in both NPs was significantly impacted by laser radiation. Phototoxicity and dark toxicity tests demonstrated that the quercetin-loaded Janus NPs have excellent promise in PDT applications.

\section{Conflicts of interest}

There are no conflicts of interest.

\section{Acknowledgements}

Sincere thanks are given to Prof S. Khoei and his group in Iran University of Medical Sciences (IUMS), Tehran, Iran, for generously supporting our cell viability assay experiments.

\section{References}

1 H. Gold and N. N. Light, ACS Nano, 2010, 4, 1033-1041.

2 R. G. Mendes, A. Bachmatiuk, B. Büchner, G. Cuniberti and M. H. Rümmeli, J. Mater. Chem. B, 2013, 1, 401-428.

3 D. A. LaVan, T. McGuire and R. Langer, Nat. Biotechnol., 2003, 21, 1184-1191.

4 S. Parveen, R. Misra and S. K. Sahoo, Nanomed. Nanotechnol. Biol. Med., 2012, 8, 147-166.

5 P. Melgar-Lesmes, A. Luquero, M. Parra-Robert, A. Mora, J. Ribera, E. R. Edelman and W. Jiménez, Nano Lett., 2018, 18, 5839-5845.

6 B. Wang, X. Su, J. Liang, L. Yang, Q. Hu, X. Shan, J. Wan and Z. Hu, Mater. Sci. Eng. C, 2018, 90, 514-522.

7 A. Baek, Y. M. Baek, H. M. Kim, B. H. Jun and D. E. Kim, Bioconjug. Chem., 2018, 29, 528-537.

8 X. Zhang, J. Yin, C. Peng, W. Hu, Z. Zhu, W. Li, C. Fan and Q. Huang, Carbon, 2011, 49, 986-995.

9 L. Dedelaite, R. D. Rodriguez, E. Andriukonis, M. Hietschold, D. R. T. Zahn and A. Ramanavicius, Sens. Actuators, B, 2018, 255, 1735-1743.

10 H. Zhao, R. Ding, X. Zhao, Y. Li, L. Qu, H. Pei, L. Yildirimer, Z. Wu and W. Zhang, Drug Discovery Today, 2017, 22, 13021317.

11 S. Yang, G. Li, D. Wang, Z. Qiao and L. Qu, Sens. Actuators, B, 2017, 238, 588-595.

12 P. A. Janeesh, H. Sami, C. R. Dhanya, S. Sivakumar and A. Abraham, RSC Adv., 2014, 4, 24484-24497.

13 B. Zhang, Y. Yan, Q. Shen, D. Ma, L. Huang, X. Cai and S. Tan, Mater. Sci. Eng. C, 2017, 79, 185-190.

14 E. Masoudipour, S. Kashanian and N. Maleki, Chem. Phys. Lett., 2017, 668, 56-63.

15 A. C. Hunter and S. M. Moghimi, Polym. Chem., 2017, 8, 4151.

16 H. Priya James, R. John, A. Alex and K. R. Anoop, Acta Pharm. Sin. B, 2014, 4, 120-127.

17 L. Peng, A. Feng, S. Liu, M. Huo, T. Fang, K. Wang, Y. Wei, X. Wang and J. Yuan, ACS Appl. Mater. Interfaces, 2016, 8, 29203-29207.

18 A. Feng, J. Liang, J. Ji, J. Dou, S. Wang and J. Yuan, Sci. Rep., 2016, 6, 1-10.

19 M. R. Islam, J. Irvine and M. J. Serpe, ACS Appl. Mater. Interfaces, 2015, 7, 24370-24376. 
20 Y. Zhan, M. Gonçalves, P. Yi, D. Capelo, Y. Zhang, J. Rodrigues, C. Liu, H. Tomás, Y. Li and P. He, J. Mater. Chem. B, 2015, 3, 4221-4230.

21 G. Fundueanu, M. Constantin, S. Bucatariu and P. Ascenzi, Macromol. Chem. Phys., 2016, 217, 2525-2533.

22 F. Liang, C. Zhang and Z. Yang, Adv. Mater., 2014, 26, 69446949.

23 H. Wu, W. Yi, Z. Chen, H. Wang and Q. Du, Carbon, 2015, 93, 473-483.

24 X. Fan, J. Yang, X. J. Loh and Z. Li, Macromol. Rapid Commun., 2018, 1800203.

25 X. Wang, X. Feng, G. Ma, L. Yao and M. Ge, Adv. Mater., 2016, 28, 3131-3137.

26 Y. Geng, P. Zhang, Q. Wang, Y. Liu and K. Pan, J. Mater. Chem. B, 2017, 5, 5390-5396.

27 J. Zhang, X. Zheng, X. Hu and Z. Xie, J. Mater. Chem. B, 2017, $5,4470-4477$.

28 N. Malatesti, I. Munitic and I. Jurak, Biophys. Rev., 2017, 9, 149-168.

29 Y. Que, Y. Liu, W. Tan, C. Feng, P. Shi, Y. Li and H. Xiaoyu, ACS Macro Lett., 2016, 5, 168-173.

30 L. Huang, Z. Li, Y. Zhao, Y. Zhang, S. Wu, J. Zhao and G. Han, J. Am. Chem. Soc., 2016, 138, 14586-14591.

31 S. Y. Li, H. Cheng, W. X. Qiu, L. H. Liu, S. Chen, Y. Hu, B. R. Xie, B. Li and X. Z. Zhang, ACS Appl. Mater. Interfaces, 2015, 7, 28319-28329.

32 C. Huang, J. Zheng, D. Ma, N. Liu, C. Zhu, J. Li and R. Yang, J. Mater. Chem. B, 2018, 6, 6424-6430.
33 D. Ma, Z. H. Liu, Q. Q. Zheng, X. Y. Zhou, Y. Zhang, Y. F. Shi, J. T. Lin and W. Xue, Macromol. Rapid Commun., 2013, 34, 548-552.

34 Y. Li, X. Zheng, X. Zhang, S. Liu, Q. Pei, M. Zheng and Z. Xie, Adv. Healthcare Mater., 2017, 6, 1-6.

35 D. C. Marcano, D. V. Kosynkin, J. M. Berlin, A. Sinitskii, Z. Sun, A. Slesarev, L. B. Alemany, W. Lu and J. M. Tour, ACS Nano, 2010, 4, 4806-4814.

36 C. Houga, J. F. Le Meins, R. Borsali, D. Taton and Y. Gnanou, Chem. Commun., 2007, 3063-3065.

37 S. Khoee, Y. Bagheri and A. Hashemi, Nanoscale, 2015, 7, 4134-4148.

38 F. Liu, Y. Ma, L. Xu, L. Liu and W. Zhang, Biomater. Sci., 2015, 3, 1218-1227.

39 M. Constantin, M. Cristea, P. Ascenzi and G. Fundueanu, eXPRESS Polym. Lett., 2011, 5, 839-848.

40 N. Xu, X. Huang, G. Yin, M. Bu, X. Pu and X. Chen, RSC Adv., 2018, 8, 15604-15612.

41 L. Xu, L. Liu, F. Liu, H. Cai and W. Zhang, Polym. Chem., 2015, 6, 2945-2954.

42 K. Nalampang, R. Molloy and W. Punyodom, Polym. Adv. Technol., 2007, 18, 240-248.

43 J. Zhang, B. A. Grzybowski and S. Granick, Langmuir, 2017, 33, 6964-6977.

44 S. Khoee and M. R. Karimi, Polymer, 2018, 142, 80-98.

45 S. Sua, J. Wang, J. Wei, R. Martínez-Zaguilán, J. Qiu and S. Wang, New J. Chem., 2015, 39, 5743-5749.

46 M. A. Taylor, F. Khathayer and S. K. Ray, Neurochem. Res., 2019, 1-11. 